\section{Comment}

I do not know of a department providing an EPS which fulfils all of these specifications for an ideal service. Nevertheless, some manage to offer something quite close to it, despite less than ideal circumstances. I do not think any of the things which I have suggested are unduly extravagant. Indeed, relative to the cost of providing adequate facilities for dealing with acute trauma or medical emergencies, the funding required for most of what $I$ have put for- ward is probably relatively modest. Part of the problem is, perhaps, this. The facilities needed for good acute medical care seem fairly clear-cut. There is little difficulty in justifying the provision of resuscitation rooms, ECG monitors, cardiac arrest teams and so on. In contrast, just what it is that is required to provide good emergency psychiatric care may be more elusive. I hope that I have been able to draw attention to some of the practical requirements for a satisfactory service.

\title{
Patients at a Psychiatric Walk-in Clinic - Who, How, Why and When
}

Camilla Haw, Senior Registrar, Regional Secure Unit, St Bernard's Hospital, Uxbridge Road, Southall, Middlesex, (formerly Registrar, Maudsley Hospital); Colin Lanceley, Senior Registrar, St Augustine's Hospital, Chartham, Canterbury, Kent (formerly locum Senior Registrar, Maudsley Hospital) and STEVEn Vickers, Research Assistant, Department of Computing, Imperial College, 180 Queen's Gate, London, SW7

In recent years there has been increasing emphasis on community-based psychiatric services. If these facilities are to be comprehensive they must include ways of assessing and managing crises and avoiding unnecessary hospitalisation. One way of meeting these needs is to provide small walk-in clinics in the community. A number of clinics have been established and more are planned. Although clients may refer themselves, access is usually limited to office hours and often only to a few hours each day.

In contrast to these small local facilities, there exist a few larger psychiatric walk-in clinics which operate a 24 hour service for all patients regardless of catchment area. One such unit is the Maudsley Emergency Clinic, though recently its 24 hour operation has been threatened by Health Service cuts. The organisation of the clinic and some of the demographic characteristics of its clientele have already been described. ${ }^{1,2,3}$

Our study aimed to answer a number of questions-is a 24 hour clinic an expensive luxury? When do people present to the clinic? Are they sicker at night? How far do people travel for help? Are clients good judges of their need for psychiatric help? Are GPS better judges? Do people abuse the clinic?

\section{The study}

All visits to the clinic during July and August 1985 were studied. For each visit the following information was derived from the clinic's log book and stored on computer: name, age, sex, date and time of arrival, referral source, whether the patient lived within the Maudsley's catchment area, distance from the Maudsley to the patient's home, diagnosis-derived from ICD-9 (where several diagnoses were given the one most relevant to the presenting problem was taken), management plan, e.g. admission, 'guesting' overnight for review the following morning, out-patient referral, follow-up appointment at the clinic, discharge. Where details were missing the case notes were studied. By arrangement, the Emergency Clinic also sees the psychiatric patients who present to King's College Casualty Department out of hours or who arrive in office hours but without a referral letter. These presentations to King's constituted $14 \%$ of the index visits during the study period.

Statistical tests. Proportions of populations were compared using the normal approximation to the binomial distribution where sample sizes were permitted, or in some cases using the binomial distribution directly.

Who uses the clinic? 650 patients made 1,050 visits during the study period. $56 \%$ were men. Mean age was 36.7 years (s.d. 14.3). Drunks were included and assumed to be suffering from alcoholism. They were not otherwise included in the analysis, except under the timing of visits. This is because it is the clinic's policy not to interview drunk patients and so no details, other than their name and arrival time, were recorded.

Schizophrenia was the most frequent diagnosis $(21 \%)$ and psychotic patients were commoner than neurotic ones $(36 \% \vee 23 \%)$. Neurotic and psychotic depression constituted $27 \%$ of cases. 
How much is the clinic used outside office hours? When follow-up visits were excluded, the clinic was nearly as busy (as judged by the numbers of new and unexpected patients arriving each hour) in the evening as during office hours (Fig. 1). Follow-up appointments were made for a morning or afternoon on a specific weekday and almost all were kept in office hours.

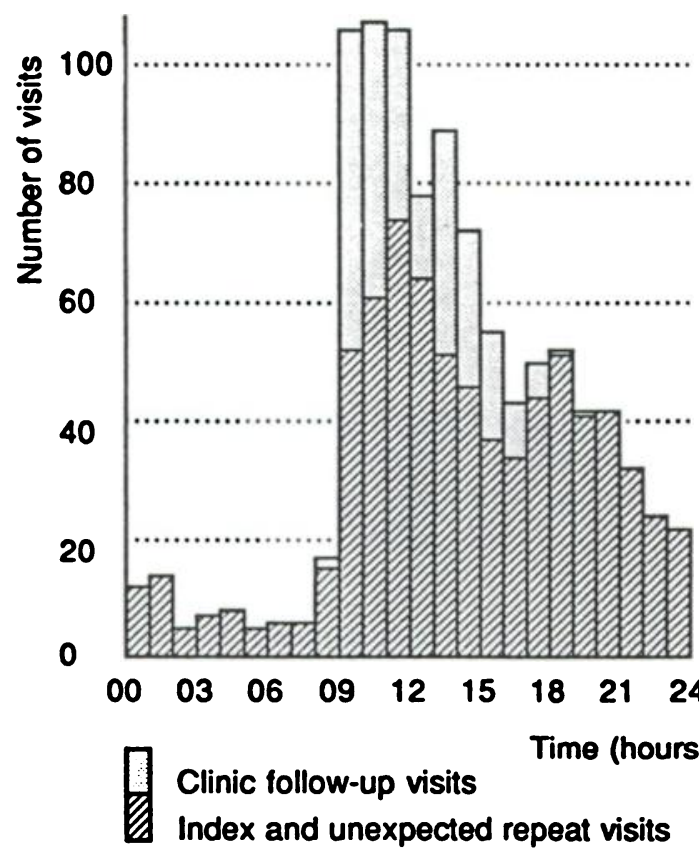

FIG. 1. Visits by time of arrival. An index visit is a patient's first visit recorded during the study period.

Who uses the clinic outside office hours? Our clinical impression was that proportionally more alcoholics, schizophrenics and personality disordered patients presented out of hours. Our hypothesis was borne out for schizophrenics (Fig. 2) and for alcoholics. The proportion of schizophrenic visits rose from $22 \%$ during the day and $23 \%$ during the evening (5 p.m.-11 p.m.) to $33 \%$ overnight (11 p.m.9 a.m.). The 'nocturnal schizophrenics' were too few in number (33) for detailed analysis. They fell into two main groups: acutely disturbed individuals, often brought in by family or friends and requiring admission, and chronic selfreferred patients with seemingly trivial complaints, e.g. nightmares, requests for repeat prescriptions and vague philosophical problems, who were discharged.

There were 54 index visits (first recorded visits) by sober alcoholics and 46 visits by drunks. Taken together proportionally more alcoholics visited overnight $(23 \%)$ than during the day $(12 \%)$ or evening (12\%). Alcoholics presenting in the morning ( 9 a.m. -1 p.m.) were more likely to be sober whilst those presenting in the evening were more likely to be drunk.

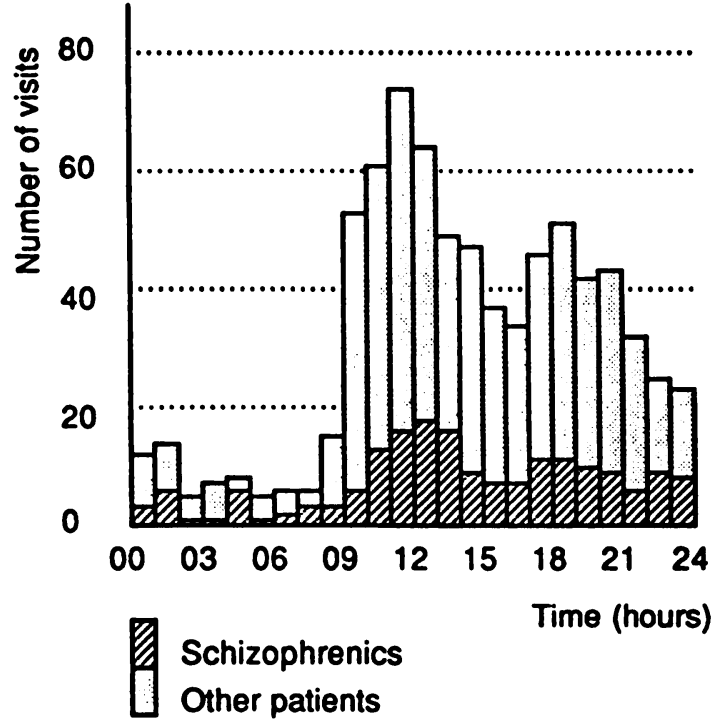

Fig. 2. Index and unexpected repeat visits by time of arrival, highlighting the visits by schizophrenic patients.

Referral source patterns. Self-referred patients were overrepresented after hours (Fig. 3). By contrast, patients referred by their GPS tended to arrive during office hours $(72 \%)$ and rarely overnight-only two. Police referrals were more evenly distributed around the clock. There were fewer GP referrals at the weekend whereas the number of police and self-referrals did not vary with the day of the week. Contrary to claims that the police sometimes abuse Section 136 , neither our experience of working in the clinic nor our statistics suggested this was true for the Camberwell police. Of the 25 Section 136 patients, $19(76 \%)$ were admitted.

Are people sicker at night? Proportionally more overnight visits $(28 \%)$ resulted in admission, compared with $18 \%$ during the day $19 \%$ during the evening (Fig. 4).

What work does the clinic do for other health authorities? The majority of patients $(59 \%)$ came from outside the catchment area. The profile of their arrival times was the same as for area patients. $66 \%$ of GP referrals were of non-area patients, perhaps indicating that GPS find the clinic provides a rapid assessment service. $66 \%$ of admissions were of nonarea patients. $45 \%$ of these non-area admissions were to the Bethlem and Maudsley, reflecting The Joint Hospitals' policy of not restricting admissions to area patients.

How far do patients travel to the clinic? $50 \%$ of patients travelled less than 1.6 miles, $80 \%$ less than 4.1 miles and $90 \%$ less than 8 miles. Although most patients lived in London, a few came long distances, e.g. the North of England, with the specific intention of being seen at the 
Maudsley. Those coming from further away tended to arrive late in the morning and early afternoon. There was no correlation between distance travelled and the likelihood of being admitted.
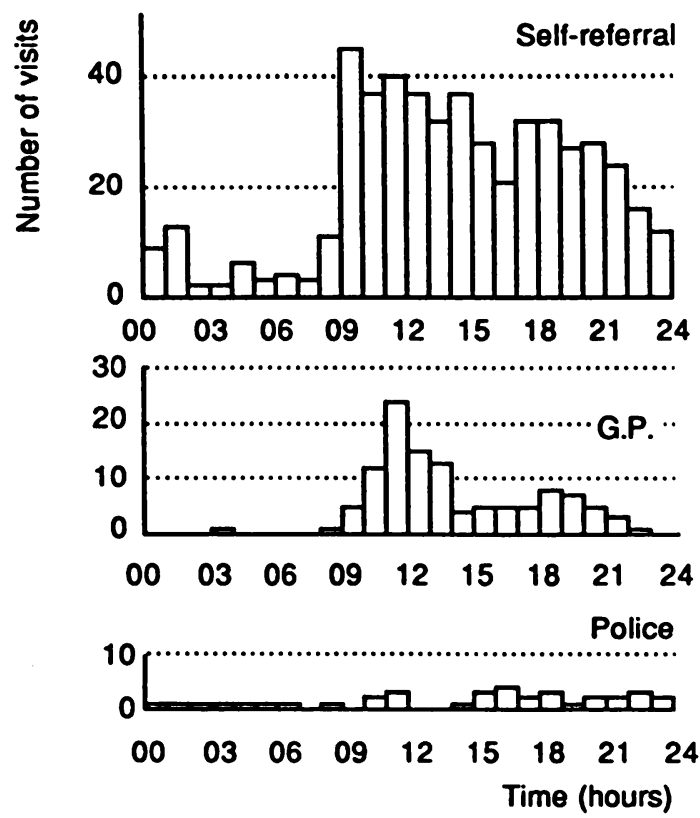

FIG. 3. Visits by time of arrival for selected referral sources.

Who is the best judge of psychiatric illness? Only six of the 355 self-referrals were thought not to be psychiatrically ill. However, we found a lower admission rate among selfreferrals $(14 \%)$ compared to GP referrals $(26 \%)$. Selfreferrals were more likely to be discharged at index visit than GP referrals $(22 \%$ v $15 \%)$, also indicating they were not as ill. Thus, self-referrals suffered from genuine psychiatric disorders but tended to have milder forms than those referred by other agencies. Patients with anxiety neurosis, personality disorder or alcoholism were more likely to be self-referred $(83 \%, 80 \%$ and $80 \%$ respectively) and these diagnoses were among the lowest admission rates of $0 \%$, $6 \%$ and $7.4 \%$ respectively.

In 1971 Kaeser \& Cooper ${ }^{4}$ looked at the reasons GPS referred patients to the Emergency Clinic rather than to the out-patient department. In general, urgent problems, e.g. behavioural disturbance, serious social difficulties or possible suicidal risk, were sent to the Emergency Clinic and non-urgent problems to out-patients. They found that in $45 \%$ of cases GPS referred patients to the Emergency Clinic with a view to admission or possible admission. However, when a GP expected a patient to be admitted, the psychiatrist agreed in only $50 \%$ of cases.

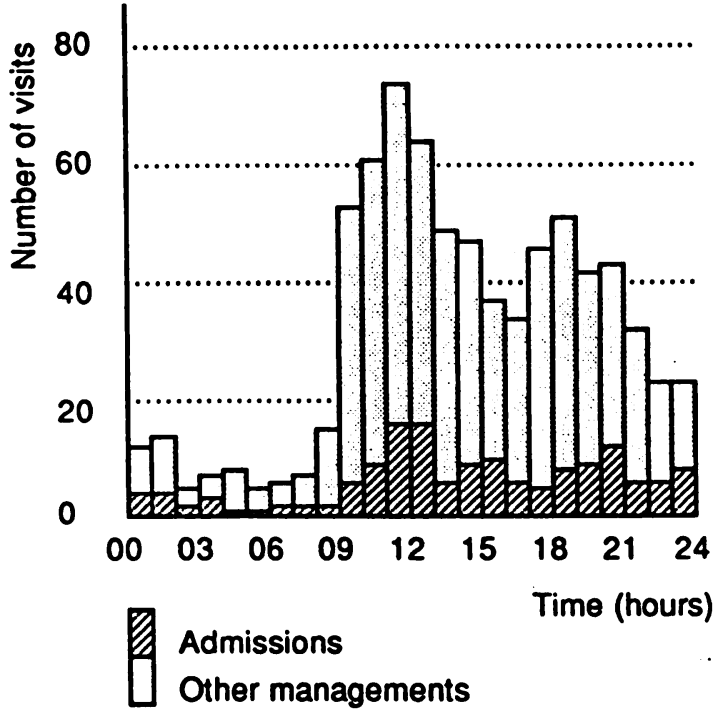

FIG. 4. Index and unexpected repeat visits by time of arrival, highlighting the visits that resulted in admissions.

Is the clinic abused? Our impression was that most people used the clinic because they had genuine difficulties. However, the service was abused by a minority: $4.4 \%$ of all visits were drunks who were sometimes disruptive and upsetting to other patients.

$31 \%$ of patients who had been discharged at index visit subsequently re-presented, in the majority of cases only to be discharged again. These 'repeaters' constituted $6 \%$ of all patients and schizophrenics (36\%), and personality disorders $(33 \%)$ were over-represented among them. Ten patients $(1.6 \%$ of the total) repeatedly re-referred themselves, two to five times during the two months of the study, only to be re-discharged at each visit. The majority were personality disordered.

Has the clientele changed over the years? The number of new visitors to the clinic each year continues to rise (Maudsley Hospital records) despite the development in London of community-based facilities.

The relative and absolute numbers of GP referrals have fallen over the years while the numbers of self-referrals have increased. A greater proportion of GP referrals resulted in admission (26\% v 10\%). Perhaps GPS have become more knowledgeable about psychiatry and reserve referral to the more disturbed patients. Many younger GPs have had psychiatric experience during their training and are better judges of when to seek specialist advice.

The proportion of men has risen since Lim's study of $1983^{3}$ from $49 \%$ to $56 \%$. This is explicable in terms of the increase in self-referrals as these tend to be male. Men seem reluctant to discuss emotional difficulties with those close to them, preferring the anonymity of a walk-in clinic and often making a sudden decision to seek help. 
Comment

Walk-in clinics are valued by clients as people can refer themselves and help and advice are readily available. Outpatient referral, a domiciliary visit by a consultant psychiatrist or a visit by a crisis intervention team each have disadvantages. They require the GP to refer, new outpatients may have to wait several weeks or months to be seen and visiting at home is not always merited or wanted by the patient.

The nature of psychiatric emergencies often reflects social and interpersonal crises rather than medical type emergencies. Studies in the USA show increasing use of emergency facilities by chronic patients, many of them suffering from psychotic disorders and lacking social supports. ${ }^{5}$ Most psychiatric emergencies are not acute crises but chronic patients struggling with long-standing problems of living.

What service implications does our study generate for the community mental health centres? At present, these walk-in clinics are used by a very different clientele, though perhaps in time this will change. The Handen Road Mental Health Resource Centre, located less than five miles away, sees more adjustment reactions and fewer schizophrenics and most patients are referred by their GPS rather than themselves. ${ }^{6}$ However, evening clinics would be valued by clients as judged by our experience. Unlike the Maudsley, local clinics would not see sufficient referrals to justify an overnight service. Arguably the accident and emergency departments of general hospitals already provide such a service, albeit in highly unsuitable surroundings and with an absence of psychiatrically trained nursing staff.

Could our 'out of hours' patients have waited until the following morning? In many cases the answer would appear to be 'yes' as acutely disturbed patients were not that common. However, it is easy for a psychiatrist to judge a problem as being non-urgent. A patient faced with distressing symptoms or worries may disagree.

The differing nature of the clientele presenting after hours is of note. $\mathrm{Lim}^{3}$ first reported the greater proportion of psychotic patients presenting to the clinic after hours. Our observation was of an excess of schizophrenic patients arriving overnight. Others have noted that $42 \%$ of 'out of hours' referrals to psychiatric social workers were psychotic of whom half required admission. ${ }^{7}$ Our experience of the 'nocturnal schizophrenic' was not always of the acutely ill individual. Half of these patients had no acute symptoms and their presentation at night appeared to relate to their eccentric, non-conformist life-styles.
The problem population of patients who make frequent use of emergency facilities despite discharge at each visit was small $(1.6 \%$ in our study). The most habitual 'repeaters' were the personality disordered and this is similar to the findings of the North America studies of patients who repeatedly visit emergency rooms.

The Maudsley Emergency Clinic attempts to meet the needs of anyone who presents to it at any time. It is able to offer short-term follow-up to clients or refer them on to a wider range of treatments available at the hospital. The clinic provides a wealth of research material for the Maudsley and Institute of Psychiatry, as well as valuable experience for the registrar, all of whom take turns to man the clinic after hours. However, use of the clinic by noncatchment area patients is not always helpful for either patients or professionals. This is particularly so when local knowledge is needed, the illness is chronic and the district services have previously been involved. Perhaps only when walk-in clinics are more widely available will this problem be resolved.

\section{ACKNOWLEDGEMENT}

We wish to thank Dr Joseph Connelly for his help and encouragement.

\section{REFERENCES}

'BRoTHWOOD, J. (1965) The work of a psychiatric emergency clinic. British Journal of Psychiatry, 111, 631-634.

${ }^{2}$ Mindham, R. H. S., Kelleher, M. J. \& Birley, J. L. T. (1973) A psychiatric casualty department. Lancet, ii, 1169-1171.

${ }^{3}$ LIM, M. H. (1983) A psychiatric emergency clinic: a study over six months. British Journal of Psychiatry, 143, 460-466.

${ }^{4}$ KAeser, A. C. \& CoOper, B. (1971) The psychiatrist patient, the general practitioner and the out-patient clinic: an operational study and a review. Psychological Medicine, 1, 312-325.

${ }^{5}$ NuRIUS, P. S. (1983) Emergency psychiatric services: a study of changing utilisation patterns and issues. International Journal of Psychiatry in Medicine, 13, 239-254.

${ }^{6}$ BOURAS, N. (1982) Mental health advice centre: three years of experience. Research report no. 1, Lewisham \& North Southwark Health Authority.

${ }^{7}$ Mountney, G. H., Fryers, T. \& Freeman, H. L. (1969) Psychiatric emergencies in an urban borough. British Medical Journal, 1, 498-500.

\section{Retirement of Dr John Evans}

Dr John Evans, FRCPsych, consultant psychiatrist at the Young People's Unit, Royal Edinburgh Hospital, is retiring from the NHS in October. He was responsible for initiating the YPU and has been its Director since its inception in 1966. 